\title{
Numerical Solution of the Aerosol Condensation/Evaporation Equation
}

\author{
Khoi Nguyen and Donald Dabdub \\ Department of Mechanical \& Aerospace Engineering \\ University of California, Irvine \\ Irvine, CA 92612, USA \\ ddabdub@uci.edu
}

\begin{abstract}
A new method is developed to solve the condensation equation as it relates to Air Quality Models using both semi-Lagrangian and Lagrangian fluxes to increase resolution and perform accurately under stringent conditions that occur in the atmosphere. The new method, Partitioned Flux Integrated Semi-Lagrangian Method (PFISLM), can be used with lower-order interpolators and they produce highly accurate results. PFISLM is positive definite, peak retentive, mass conservative, and suppresses oscillations. Research indicates the differences between PFISLM and other traditional flux integrated semi-Lagrangian methods are significant when solving the aerosol condensation/evaporation equation. PFISLM is created to handle specific difficulties associated with the time and space discretization of the aerosol operator in air quality models.
\end{abstract}

\section{Introduction}

Air quality models that include aerosol dynamics often employ the continuous distribution approach (Pilinis, 1990) to represent aerosols undergoing evaporation and condensation. The fundamental equation governing the condensation process for an internally mixed aerosol is derived in Pilinis (1990). The equation is given as

$$
\frac{\partial p_{i}}{\partial t}=H_{i} p-\frac{1}{3} \frac{\partial H p_{i}}{\partial \mu},
$$

where $p_{i}, H_{i}, \mu, p=\sum_{i}^{n} p_{i}$, and $H=\sum_{i}^{n} H_{i}$ are the mass distribution of species $i$, mass transfer rate of species $i, \log$ of the diameter of the particle, total concentrations, and total mass transfer rates respectively with $n$ being the number of aerosol compounds.

Typically, Equation (1) is solved by means of operator splitting into two parts: the growth,

$$
\frac{\partial p_{i}}{\partial t}=H_{i} p
$$

and the redistribution,

$$
\frac{\partial p_{i}}{\partial t}=-\frac{1}{3} \frac{\partial H p_{i}}{\partial \mu}
$$

V.N. Alexandrov et al. (Eds.): ICCS 2001, LNCS 2074, pp. 77-81 2001.

(C) Springer-Verlag Berlin Heidelberg 2001 
Section 2 describes a new algorithm to solve the aerosol condensation/evaporation equation. Numerical tests are presented in Section 3.

\section{The Algorithm}

\subsection{Aerosol Redistribution}

PFISLM uses both semi-Lagrangian fluxes and Lagrangian positions to resolve the space discretization. A graphical representation of the new scheme is shown in Figure 1. A typical semi-Lagrangian flux is the mass that is contained from the semi-Lagrangian position of the cell interface, $\left(\mu_{1}\right)$ to the cell interface, $\left(\mu_{2}\right)$.
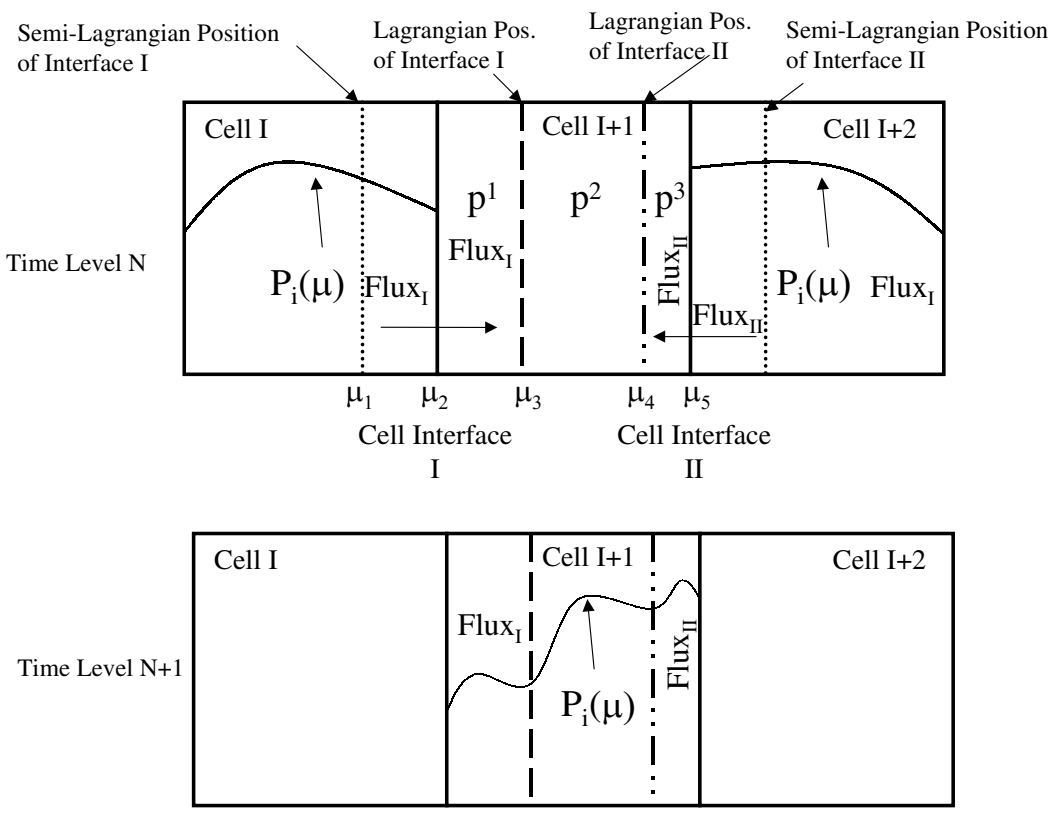

Fig. 1. Partitioned Flux Integrated Semi-Lagrangian Method uses both the SemiLagrangian and Lagrangian position of cell interfaces in its computation. The flux leaving cell interface $\mathrm{I}$ is not averaged into cell $\mathrm{I}+1$ but is recorded into the partition defined from $\mu_{2}$ and $\mu_{3}$.

The evacuated semi-Lagrangian flux enters the partition defined by the cell interface, $\left(\mu_{2}\right)$ and the Lagrangian position, $\left(\mu_{3}\right)$. This area from $\left(\mu_{2}\right)$ to $\left(\mu_{3}\right)$ 
is known as a partition. Since, the total mass in cell $I+1$ is known, the distribution inside each cell is further resolved. Namely, the mass occupied by the first partition, $\mu_{2}$ to $\mu_{3}$, is $p^{1}=F_{I}$, the mass occupied by the second partition, $\mu_{3}$ to $\mu_{4}$, is $p^{2}=p_{i}(t)$, and the mass occupied by the third partition from $\mu_{4}$ to $\mu_{5}$ is $p^{3}=F_{I I}$. In the next time step, the mass distribution inside cell $I+1$ is interpolated (using both linear and quadratic interpolators) more accurately because more information is available to describe the mass partitioning inside the cell due to the Lagrangian partitioning. Depending on the mass transfer rate, each cell can have up to three partitions.

\section{$2.2 \quad$ Aerosol Growth}

The growth term given in Equation (2), is computed from a mass balance and the growth law. The growth is computed for the entire cell and is distributed to the enclosed partitions accordingly. The growth for the entire cell is found by a direct integration of the growth equations,

$$
\begin{gathered}
\frac{\partial p_{i}}{\partial t}=H_{i} p \\
\frac{\partial p}{\partial t}=H p .
\end{gathered}
$$

The solution to those two simultaneous equations is given as

$$
p_{i}(t+\Delta t, \mu)=p_{i}(t, \mu)+\frac{H_{i}}{H} p(t, \mu)\left[e^{H \Delta t}-1\right] .
$$

The mass increase or decrease in each partition is computed by solving a simple set of equations that represents mass conservation, the solution to the growth equation, and the contribution of the cell growth from each partition in accordance to its relative mass to the entire cell. That set of equations is given as

$$
\begin{gathered}
p_{i} \Delta \mu=p^{1} \Delta \mu_{1}+p^{2} \Delta \mu_{2}+p^{3} \Delta \mu_{3} \\
\text { growth }=\frac{H_{i}}{H} p(t, \mu)\left[e^{H \Delta t}-1\right] \\
\text { massgrowth }_{j}=\text { massgrowth }_{\text {mass }} \\
\text { mass }
\end{gathered}
$$

Here the massgrowth is the growth of the mass in the entire cell, growth is the growth of concentration in the entire cell, mass $s_{j}$ is the mass occupied by partition $j$, massgrowt $h_{j}$ is the growth of the mass in partition $j, p_{i}$ is the concentration in the entire cell, and $p^{j}$ is the concentration in partition $j$. Substituting massgrowt $_{j}=$ growt $_{j} \Delta \mu_{j}$, massgrowth $=$ growth $\Delta \mu$, and $\operatorname{mass}_{j}=p^{j} \Delta \mu_{j}$ into Equation (12), the solution to the growth term in each partition is obtained as,

$$
\operatorname{growth}_{j}=\operatorname{growth} \frac{p^{j}}{p_{i}} .
$$




\section{Numerical Test}

There were hundreds of test perform in order to gauge the accuracy of the solver. For the sake of brevity, here we will report on one of them. The test consists on 200 iterations of back and forth condensation and evaporations of a given aerosol distribution using 12 fixed bins. The rate of evaporation and condensations is constant. Therefore, the final solution is expected to equal the initial conditions. Figure 2 shows that results are significantly less diffusive than those produced by Bott's solver (Bott, 1989) which is currently used in several air quality models.

The numerical test uses the following initial conditions:

$$
p_{i}(\mu)= \begin{cases}\frac{i}{20}\left(1+\cos \left[\frac{\mu-5}{4}\right]\right) & \text { if }|\mu-5| \leq 4 \\ 0 & \text { otherwise }\end{cases}
$$

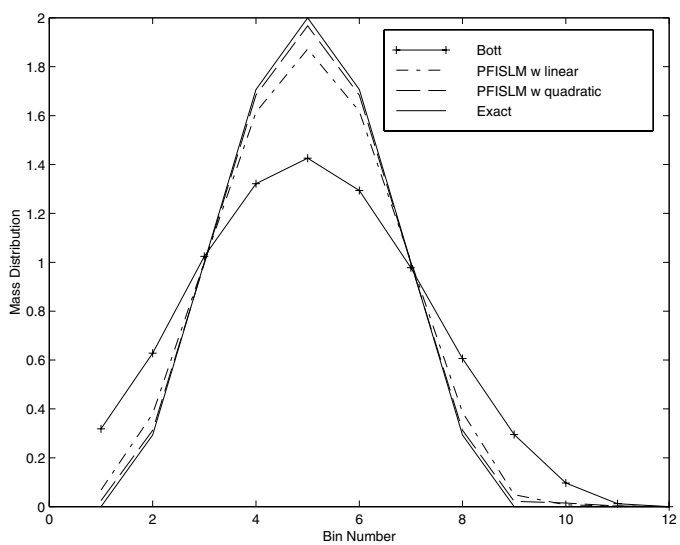

Fig. 2. Numerical tests show that the Bott solver is diffusive while the PFISLM with linear and quadratic interpolators preserve the peaks within $10 \%$.

\section{Conclusions}

A new approach to solving the condensation equation is developed to handle these difficult characteristics of the aerosol operator. The new method is mass conservative, positive definite, peak retentive, and suppresses oscillations. The approach is a super set of flux integrated semi-Lagrangian methods using both semi-Lagrangian and Lagrangian fluxes. It effectively increases the resolution of the bins by keeping information about the partitions inside each bin. This increased resolution permits accurate representation of the actual dynamics of aerosols, especially with limited number of bins. 


\section{References}

Pilinis, C.: Derivation and numerical solution of the species mass distribution equations for multicomponent particulate systems. Atmos. Environ. 24 (1990) 1923-1928

Bott, A.: A positive definite advection scheme obtained by nonlinear renormalization of the advective fluxes. Mon. Wea. Rev. 117 (1989) 1006-1015 